\title{
Manufacture of Input Support Device for Livelihood Support of ALS Patients
}

\author{
Yuki Fukuda $^{\mathrm{a}}$, Yutaka Tange ${ }^{\mathrm{a}}$ \\ ${ }^{a}$ National Institute of Technology, Maizuru College, Maizuru 625-8511, JAPAN \\ Corresponding Author : a0386@g.maizuru-ct.ac.jp
}

\begin{abstract}
In order to develop an input interface with the ALS (amyotrophic lateral sclerosis) patients, we prepared an input support device using the eye potential generated during eye movement. The cornea and the retina are charged with positive and negative potentials respectively, and eye potential can be acquired by electrodes on the temple, earlobe, and forehead. You can obtain EOG (electrooculogram) waveforms by measuring the obtained eye potential with a nystagmograph. EOG waveforms are effective for identifying waveforms distinctive of directions of eye movement and judging which ways eyeballs move. Using the data obtained from this EOG waveforms to input into an electric wheelchair, we will develop a livelihood support system for ALS patients.
\end{abstract}

Keywords: Eye Potential, ALS, Livelihood Support System, Input Support Device.

\section{Introduction}

\subsection{The Present Situation of ALS}

At present, there are about 10,000 patients with ALS (amyotrophic lateral sclerosis) in Japan, and the number is growing ${ }^{(1)}$. Fig. 1 shows the transition of the number of ALS patients by fiscal year. ALS is a disease that the motor nervous system gradually gets senile and hard to work. Although the extent and the progression of the motor nervous system disorder vary among individual patients, they are not classified as people with a sensory nerve disorder. However, it is often difficult for them to communicate in conversation due to a decline in muscular strength. Even in such a case,

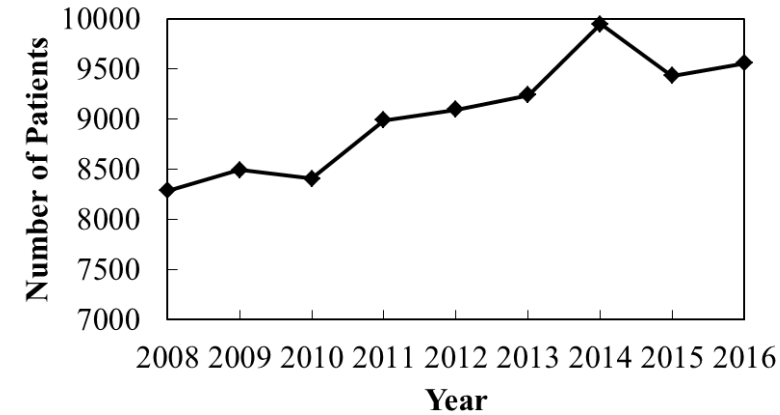

Fig. 1. Transition of the number of ALS patients by fiscal year

a method of supporting ALS patients by using other remaining motor nervous systems has been considered. For example, there is a communication support device using muscles of facial expression ${ }^{(2)}$. As the disease progresses, however, the performance of muscles of facial expression decreases, which makes it impossible to use the device.

\section{$1.2 \quad$ Eye-gaze Input}

There are different ways of eye-gaze input as shown in Fig. 2. The VOG (videooculogram) method in which you detect eye movement in a video, the corneal reflex method in which you detect it by the reflected light of infrared rays to irradiate the cornea with, the scleral reflex method for detecting eye movement based on the difference of reflectance between the iris and the white of the eye, and the EOG (electrooculogram) method for detecting eye movement by waveforms coming from the potential difference between the cornea and the retina ${ }^{(3)}$. Among them, the VOG $\operatorname{method}^{(4)}$, the corneal reflex method, and the scleral reflex method always require goggles and, moreover, a computer with high processing capability that enables us to process image in real time. 


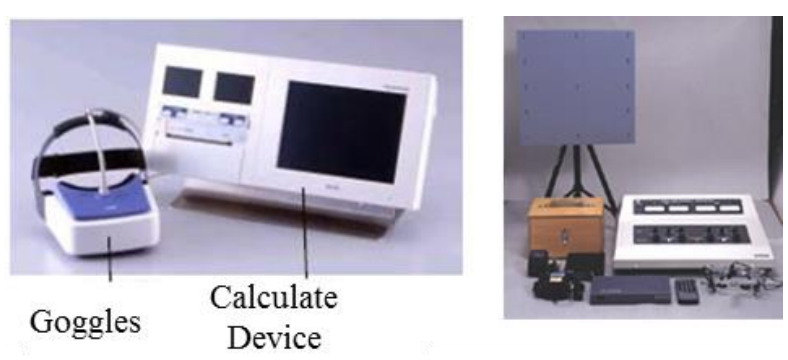

(a) VOG method ${ }^{(4)}$ (b) Scleral reflection method ${ }^{(5)}$ Fig. 2. Examples of devices that measure eye movements

In addition, the corneal and the scleral reflex $\operatorname{methods}^{(5)}$ are difficult to use for a long time due to eye fatigue, and the accessory device is about $7 \mathrm{~kg}$, which is too heavy to carry about. The EOG method requires precision to measure eye movement precisely.

In this research, we focused on the EOG method, which is suitable for miniaturization and leads to less burden on subjects. In the method, eye movement can be detected with small electrodes on the face without depending on the environment in which it is used. Also, since the assisting device only needs the information about directions of eye movement, it is not necessary to detect eye movement with high accuracy.

\subsection{The Purpose of This Research}

In this research, we focus on eye movement, which is the least influenced by the progress of ALS and allows devices to perform multiple operations. Our goal is to develop a livelihood support system for ALS patients by using EOG waveforms obtained from this eye movement as input signals to other systems.

At this stage, we think about applying the system to electric wheelchairs. For that purpose, we need to develop a nystagmograph. We improved the nystagmograph we developed in the previous research $^{(6)}$. Fig. 3 shows the structure chart of the livelihood support system proposed in this research.

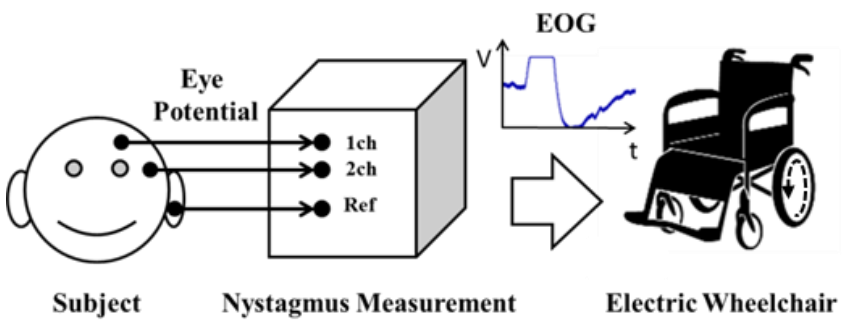

Fig. 3. Livelihood support system suggested in this research

\section{2. $\quad$ EOG}

\subsection{Generative Principle of Eye Potential}

EOG refers to an eye potential diagram, in which waveforms characterized by the directions of eye movement are observed. A positive electric potential is charged on the cornea and a negative electric potential is charged on the retina side, and therefore an eyeball can be regarded as a battery. As the cornea approaches the electrode on the skin surface, the potential rises, and conversely, as the retina approaches, the potential falls ${ }^{(7)}$. Fig. 4 shows a mechanism by which EOG waveforms change due to eye movements. To measure the horizontal movement, you paste an electrode on the right temple. To measure the vertical movement, you paste an electrode on the upper part of the right eye When the eyeball is moved to the right, the cornea approaches the electrode on the temple and the potential rises. On the other hand, when the eyeball is moved to the left, the retina approaches the electrode and the potential falls. Likewise, the potential rises in case the eyeball is moved upward, and it falls in case the eyeball is moved downward.

\subsection{Criteria for Judging}

Fig. 5 shows criteria for judging which ways the eye move. EOG waveforms can be obtained by measuring the amount of change when the potential rises or falls with a differentiator. EOG waveforms are distinctive enough to judge eye movement. The waveforms in the Fig. 5 are the results when the eye is moved up and down, left and right, and back to the front with the head fixed. The first line of the table shows the waveforms in case an electrode is pasted on the temple, and the second line in case an electrode on the upper part of the eye.

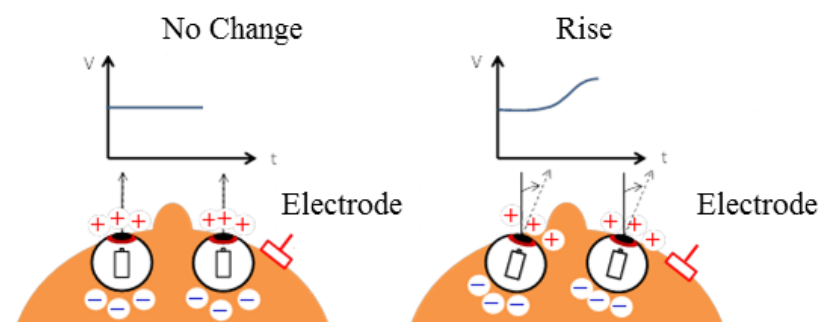

Fig. 4. Mechanism of eye potential changes 

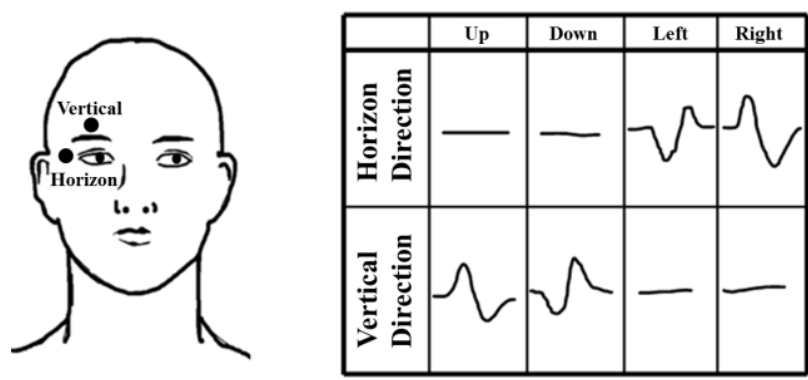

Fig. 5. Decision criteria for up and down, left and right

\section{Considering Nystagmograph}

\subsection{Block Diagram of Nystagmograph}

Fig. 6 shows the block diagram of the nystagmograph we used. Since the eye potential is very small ranging from 5 to $50[\mu \mathrm{V}]$, the signal needs to be amplified with the instrumentation amplifying circuit and the non-inverting amplifying circuit. Then, the amplified signal needs to be filtered to reduce high frequency noise with a low pass filter (cut off frequency $3.38[\mathrm{~Hz}]$ ) and a differentiator. In order to hold baseline fluctuation, the DC servo circuit and the virtual GND are introduced. The voltage of the virtual GND was set to $2.5[\mathrm{~V}]$.

\subsection{Electrode}

There are many types of electrodes, each of which is distinctive. Saturated calomel electrodes use mercury, which is harmful to the human body. Reversible hydrogen electrodes are suitable for measuring liquid, not the human body. Dish electrodes are harder to change in potential than other electrodes. Moreover, they can be repeatedly used. However, it is necessary to apply a conductive paste between the electrode and the skin. It is a problem that waveforms are affected by the amount of conductive paste. On the other hand, disposable electrodes can be little affected by the amount of conductive paste, but they can't be repeatedly used. Judging from these characteristics, we used a dish electrode and a disposable electrode in this research. Fig. 7 (a) and (b) show the dish electrode and the disposable electrode we used.

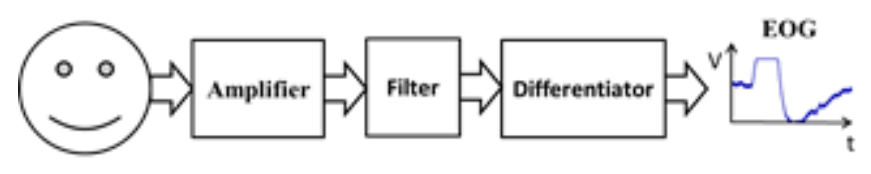

Fig. 6. Configuration diagram of the manufactured device for measuring eye potential

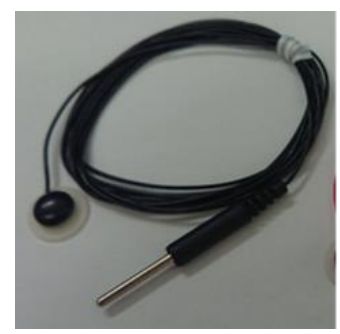

(a) Dish electrode

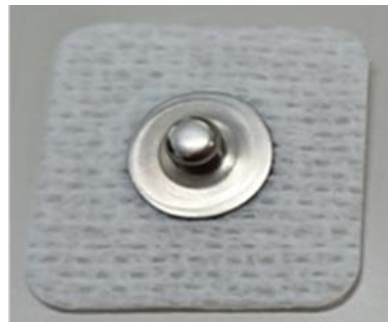

(b) Disposable electrode Fig. 7. Disposable electrode

\section{Measurement of Eye Movement}

\subsection{Measuring Method}

In the experiment, we measured the horizontal and the vertical movements of the right eye with a dish electrode. We set the reference potential on the earlobe and measured with the reference voltage $2.5[\mathrm{~V}]$. The subject is a 20 -year old male. As stated above, we put an electrode on the right temple to measure the horizontal movement and on the upper part of the right eye to measure the vertical movement. Prior to conducting this experiment, we got the subject's approval. Fig. 8 shows the position where we put electrodes.

\subsection{Measurements}

Fig. 9 shows EOG waveforms as measurements. A distinctive EOG waveform can be seen around 5 seconds. In addition, when there is no eye movement, it is going up and down slightly near the reference potential of 2.5 [V]. This may be a myoelectric potential due to facial muscles. This myoelectric potential is a small output compared with the eye potential. We can neglect it. In considering the validity of the measurements, we confirmed that the characteristics of the waveforms accorded with the criteria in Fig. 5. This shows that the measurements are valid. 


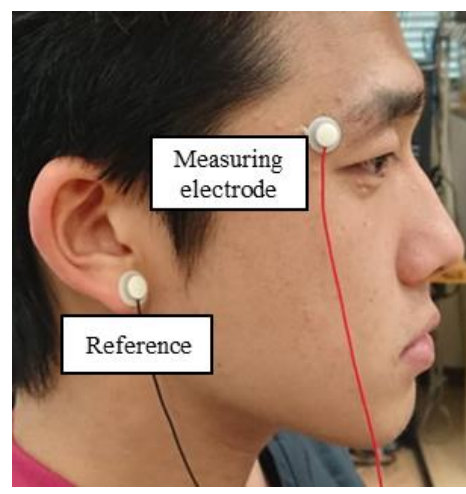

Fig. 8. The electrode pasting position

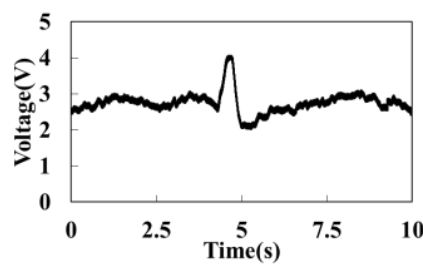

(a) Move up

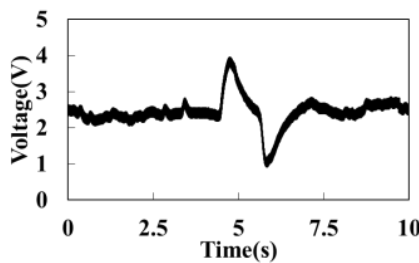

(c) Move light

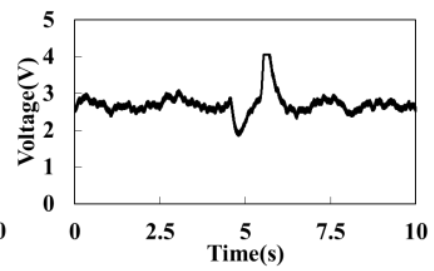

(b) Move down

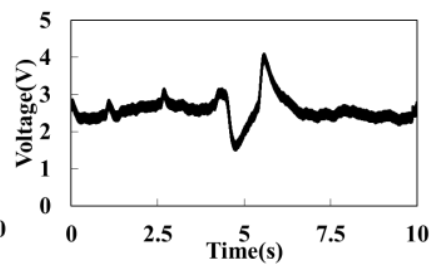

(d) Move left
Fig. 9. Measurement result of the right eye potential

\subsection{Voluntary Blink and Involuntary Blink}

When measuring the eye potential, eye movement might not be measured correctly due to the influence of voluntary blinking or involuntary blinking. Therefore, we measured voluntary blinking and involuntary blinking with the nystagmograph we developed in this research, and confirmed that they did not affect the EOG waveforms.

We measured with a measuring electrode on the right temple and a reference electrode on the earlobe. The subject was a 21-year old healthy male, and we got his approval beforehand.

Fig. 10 (a) and (b) show the measurements of voluntary blink and involuntary blink. The peak value of voltage in voluntary blink is about 3.10 [V], and the one in involuntary blink is about 2.75 [V]. Since the waveforms are smaller than the EOG waveforms in Fig. 9 , it is possible to measure eye movement correctly without any influence of voluntary and involuntary blinks.
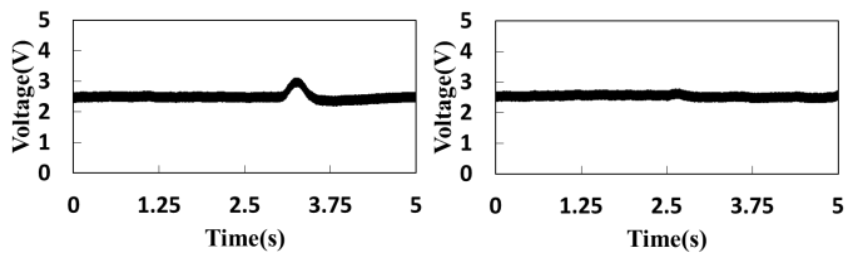

(a) Voluntary blinking (b) Involuntary blinking

Fig. 10. Measurement of blink

\subsection{Changes in Eye Potential by Electrodes}

In our laboratory, we had used dish electrodes (Nihon Kohden, NT-613) to measure the eye potential so far. As stated above, however, EOG waveforms are affected by how much we apply conductive paste. Therefore, in this research, we used disposable electrodes (B1OPAC systems, EL513), which do not require conductive paste and got EOG waveforms by pasting them on the same part as we pasted dish electrodes.

Fig. 11 shows two examples of the measurements with dish electrodes and disposable electrodes. Fig. 11 (b) shows less noise and more stable baseline than Fig. 11 (a). Judging from the results, by measuring the eye potential with disposable electrodes, EOG waveforms no longer depend on the applied amount of conductive paste. Also, it can be expected to prevent malfunctions when controlling an actual electric wheelchair.

\section{LED Control Simulating the Operation of Electric Wheelchair}

\subsection{Basic Experiment}

Electric wheelchairs are very helpful to ALS patients' moving. Therefore, assuming that ALS patients control an electric wheelchair by eye movement, we had basic tests with an LED control circuit. Since the electric wheelchair (made by YAMAHA), which we are going to use in this research, runs in 2.5 [V], we made experiments with the power supply $5[\mathrm{~V}]$ and the virtual GND $2.5[\mathrm{~V}]$.

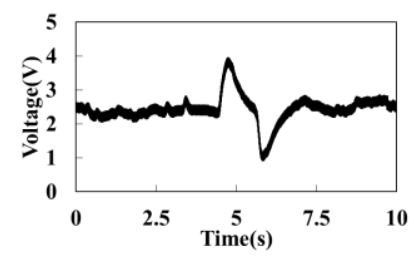

(a) Dish electrode

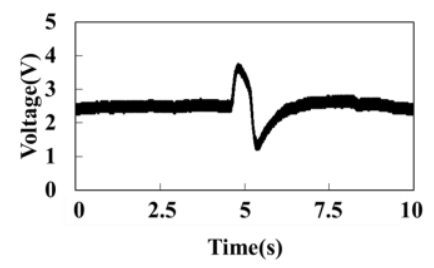

(b) Disposable electrode

Fig. 11. Measurement result of the eye potential 


\subsection{LED Control}

We tested the LED control circuit by A/D converting EOG waveforms with a PIC (Peripheral Interface Controller) microcomputer (model number:16f785) and judging eye movement. At present, we assume that an electric wheelchair will be controlled with the movement of the right eye, which is likely to be a dominant eye. We controlled the circuit to turn on the yellow LED when the right eye moved to the right and the red LED when it moved to the left. Fig. 12 shows a flowchart of the LED control.

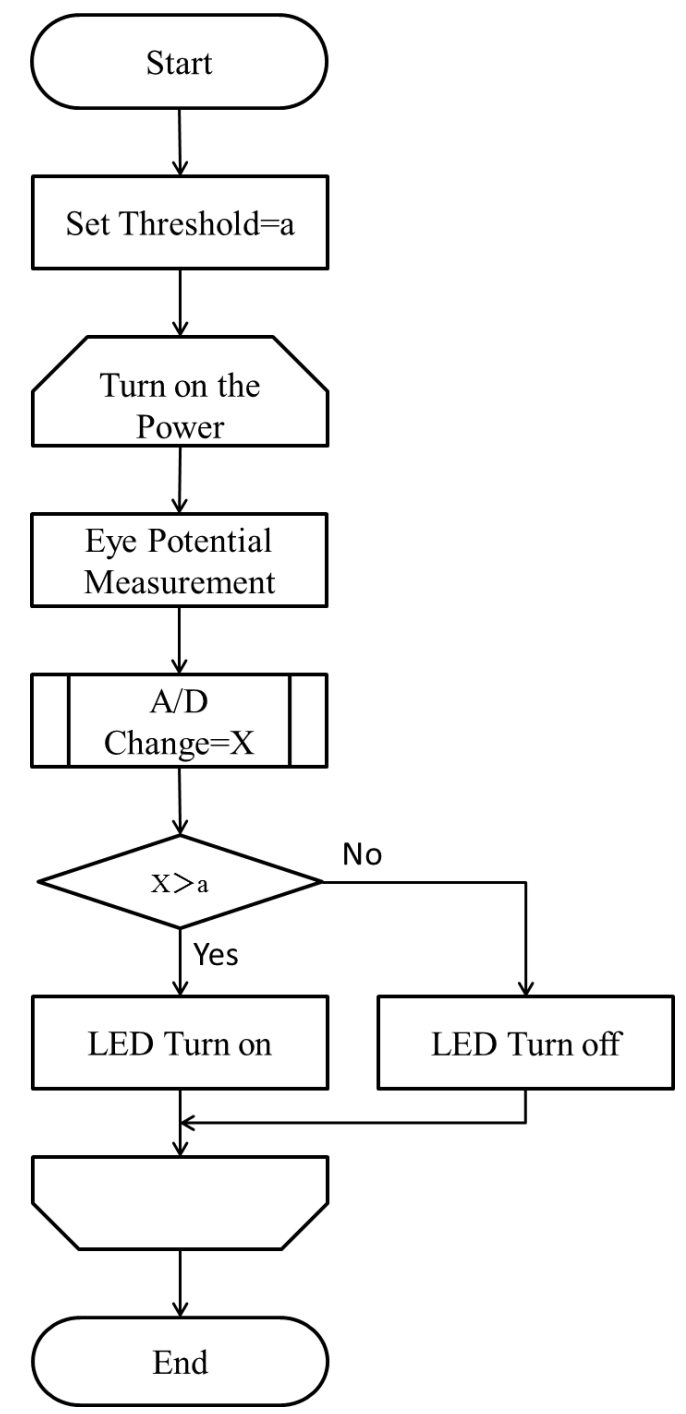

Fig. 12. Flowchart of LED control

\subsection{Result of the Experiments}

Fig. 13 shows the result of the LED control experiments. Fig. 13 (a) shows the waveform when the right eye moved to the right. At that time, we confirmed the LED turned yellow. Fig. 13 (b) shows the waveform when the right eye moved to the left. Then, we confirmed the LED turned red. Judging from the result, it is possible to control LED with EOG waveforms. By applying this result, we will develop the livelihood support system for ALS patients further.

\section{Conclusion}

In this research, we developed the livelihood support system for ALS patients by using EOG waveforms obtained from eye movement. At the present stage, we made the nystagmograph for measuring EOG waveforms without being affected by voluntary and involuntary blinks, and afterward conducted the LED control experiment simulating the operation of an electric wheelchair. Finally, we confirmed that LED can be controlled by EOG waveforms. In future plans, we will develop the livelihood support system for ALS patients by applying this system to electric wheelchairs.

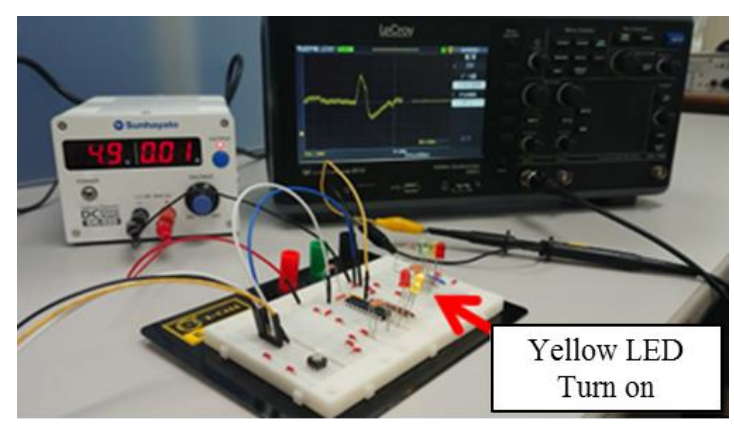

(a) Move light

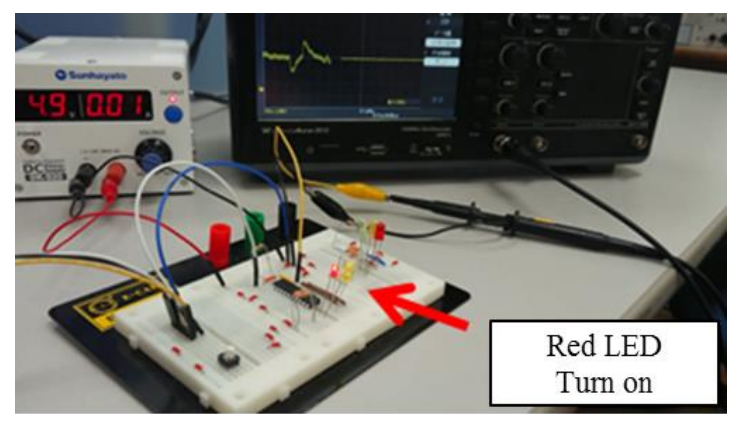

(b) Move left

Fig. 13. LED control experiment 
(1) Japan Intractable Diseases Information Center [online] http://www.nanbyou.or.jp

(2) Yuki Yamashita, Hiroki Tamura, Koichi Tanno, Takako Toyama : "A Development of the Electric Wheelchir Hands-Free Safety Control System using the s-EMG of Facial Muscles", The 23rd institute of electrical engineers of japan

(3) Shuhei Chiba, Junichi Hori : "Development of EOG-Based Character Input Interface Controlled by Three-Directional Eye Movements", The 23rd Institute of Electronics, Information and Communication Engineers Well-being Information Technology 110(53), 39-44, 2010-05-14

(4) MeditesterVOG (CD8001) - Pmda, [online] http://www.info.pmda.go.jp/ygo/pack/730056/2150 0BZZ00136000_A_01_01/

(5) Takei Scientific Instruments Co.,Ltd. [online] http://www.takei-si.co.jp/productinfo/ detail/98.html

(6) Takumi Kitajima, Yutaka Tange, Junichi Hori : "Development of Support System for Tablet-Type Device using EOG", The 35th Annual International IEEE EMBS Conference

(7) Ikuto Kazama, Junichi Hori, Yutaka Tange : "Improvement of Eye Movement Communication Aids Considering Tablet Device Operations", IEICE technical report 113(222), 1-6, 2013-09-24 